\title{
Why diagnosing inflammatory breast cancer is hard and how to overcome the challenges: a narrative review
}

\author{
Huong T. Le-Petross ${ }^{1,2}$, Wintana Balema ${ }^{2,3,4}$, Wendy A. Woodward ${ }^{2,4}$ \\ ${ }^{1}$ Department of Breast Radiology, The University of Texas MD Anderson Cancer Center, Graduate School of Biomedical Sciences, The University \\ of Texas Health Science Center at Houston, Houston, TX, USA; ${ }^{2}$ Morgan Welch IBC Clinic and Research Program, The University of Texas \\ MD Anderson Cancer Center Houston, Houston, TX, USA; ${ }^{3}$ Department of Experimental Radiation Oncology, The University of Texas MD \\ Anderson Cancer Center, Graduate School of Biomedical Sciences, The University of Texas Health Science Center at Houston, Houston, TX, \\ USA; ${ }^{4}$ Department of Radiation Oncology, The University of Texas MD Anderson Cancer Center, Graduate School of Biomedical Sciences, The \\ University of Texas Health Science Center at Houston, Houston, TX, USA \\ Contributions: (I) Conception and design: WA Woodward; (II) Administrative support: WA Woodward, W Balema; (III) Provision of study materials \\ or patients: HT Le-Petross, WA Woodward; (IV) Collection and assembly of data: All authors; (V) Data analysis and interpretation: WA Woodward, \\ HT Le-Petross; (VI) Manuscript writing: All authors; (VII) Final approval of manuscript: All authors. \\ Correspondence to: Wendy A. Woodward, MD, PhD. Department of Radiation Oncology, The University of Texas MD Anderson Cancer Center, Box \\ 1404, Houston, TX 77030, USA. Email: wwoodward@mdanderson.org.
}

Objective: The purpose of this narrative review is to summarize the contributors to misdiagnosis or delayed diagnosis of inflammatory breast cancer (IBC) and strategies for expedient diagnosis.

Background: Patients with IBC often report the disease as initially being misdiagnosed, most commonly as mastitis.

Methods: We reviewed the literature on this challenging diagnosis by using sequential PubMed search criteria including IBC breast symptoms, IBC diagnosis, and IBC imaging modalities to augment the authors' knowledge of IBC. Other references were added from the manuscripts identified in the PubMed searches and from manuscript reviewers.

Conclusions: Several factors contribute to the delayed diagnosis of IBC. One important factor is that IBC is uncommon, and many generalists may not be aware of it in the differential diagnosis of breast skin symptoms. Several features of IBC contribute to the low sensitivity of mammography for its detection, and so the diagnosis is based on clinical factors and is thereby subjective. The presentation can be highly varied; classic textbook images that do not capture the range of presenting signs and symptoms across skin tones may contribute to missed diagnoses in patients with atypical presentations. In fact, the staging system of the American Joint Committee on Cancer, which requires erythema of the breast skin for diagnosis, may exclude patients with obvious global breast skin findings that are not explicitly red. We present an adapted algorithm for working up the undiagnosed inflammatory breast to ensure the timely and accurate diagnosis of IBC. We assert that frank, non-erythematous global skin signs in an enlarged breast with diffuse breast malignancy are sufficient to diagnose IBC if the timing of these signs and findings on biopsy are consistent. We further provide images of atypical IBC identified by global breast skin signs, including peau d'orange, consistent with IBC in the absence of frank erythema.

Keywords: Inflammatory breast cancer (IBC); peau d'orange; erythema; skin thickening; redness; breast swelling; breast cancer; T4D

Submitted Aug 31, 2021. Accepted for publication Dec 09, 2021.

doi: $10.21037 /$ cco-21-116

View this article at: https://dx.doi.org/10.21037/cco-21-116 


\section{Introduction}

Inflammatory breast cancer (IBC) is a virulent subtype of invasive breast cancer that typically presents with breast skin symptoms such as erythema and edema of the breast skin. It is a crucial diagnosis to rule out when someone presents with a red or inflamed breast, but it can be a difficult diagnosis to make, both at presentation and even after a diagnosis of malignancy (1). Our objective is to review the literature highlighting factors that contribute to the difficulty of making this diagnosis and to provide updated guidance on how to avoid these challenges. Briefly, we note that the likelihood of a generalist ever seeing a case of IBC is very low, which increases the risk that it may be overlooked on the differential diagnosis for inflammatory breast symptoms. The risk of misdiagnosis is further increased by the low sensitivity of mammography, the most commonly ordered screening exam for breast lesions suspected of being malignant $(2,3)$. This means physicians who suspect cancer may be wrongly reassured by falsenegative findings on a mammogram and miss the diagnosis of IBC instead of moving on to ultrasonography, magnetic resonance imaging (MRI), and biopsy when needed (4). In addition, when malignancy is identified, it can often be misdiagnosed as non-IBC owing to subjectivity in the diagnosis of IBC, heterogeneity in presentation of IBC, and, perhaps, an overly rigid staging system requiring erythema and concurrent edema and peau d'orange to make the diagnosis (5-8). These factors greatly complicate reporting of IBC and research as well, and efforts are under way to refine the diagnosis. We present the following article in accordance with the Narrative Review reporting checklist (available at https://dx.doi.org/10.21037/cco-21-116).

\section{Methods}

References were identified by using PubMed search criteria including IBC breast symptoms, IBC diagnosis, and IBC imaging modalities to augment the authors' knowledge of IBC. Other references were added from manuscripts identified in the PubMed searches and from manuscript reviewers. References were considered relevant if they focused on making the diagnosis of IBC at the onset of symptoms; imaging patients with symptoms at the time of presentation and before diagnosis; or distinguishing IBC from non-IBC among women with symptoms and a known breast malignancy. All included references were reviewed in their entirety by at least one author.

\section{Discussion}

\section{Definitions and limits of the American foint Committee on Cancer (AFCC) staging definition}

The presentation of IBC is attributed to diffuse and rapid obstruction of lymphatics in the breast by tumor emboli, which can result in the signs and symptoms of IBC: skin edema due to direct obstruction; hyperemia related to dilation of blood vessels in lymphatic obstructed skin; and breast swelling (9). Unfortunately, the single sign that is mandatory for diagnosis of IBC in the widely used AJCC staging system is erythema, which in fact can wax and wane, or not be present at all, in spite of obvious global lymphatic obstruction leading to skin edema and breast swelling. The 8th [2017] edition of the AJCC staging manual defines IBC, stage T4D, as a "clinical-pathological entity characterized by diffuse erythema and edema involving approximately a third or more of the skin of the breast" (10). It further stipulates that the time from first symptom to diagnosis be less than 6 months, given the characteristically rapid evolution of IBC, and that a pathologic diagnosis of invasive cancer is required. The phenotype is attributed to breast and dermal lymphatics being clogged by tumor emboli; however, pathologic identification of dermal lymphatic emboli is not required for the diagnosis of IBC, and patients with dermal lymphatic emboli without clinical signs and symptoms are not considered to have IBC according to the AJCC criteria. In 2011, an international panel sought to define the minimum criteria required for the diagnosis of IBC and to refine the AJCC statements on breast skin involvement to "rapid onset of breast erythema and/or peau d'orange, and/or warm breast," with erythema occupying at least one third of the breast (5). At the very least, these variations in minimum requirements have led to some confusion and ambiguity, as was illustrated in an extensive external review of medical photographs and records of 274 patients diagnosed with IBC across six sites in Egypt, Tunisia, and Morocco (8). The diagnosis of IBC was made according to the expert panel consensus statement in $76 \%$ of the IBC cases selected for that study, and only $36 \%$ adhered to the AJCC definition. Nevertheless, $86 \%$ were confirmed as IBC by either photographic review or adherence to the consensus statement by independent, external experts. Many cases excluded by the AJCC definition in this study were excluded because careful reading suggests that both peau d'orange and edema must be present, a conclusion not supported by clinical expert review (8). 


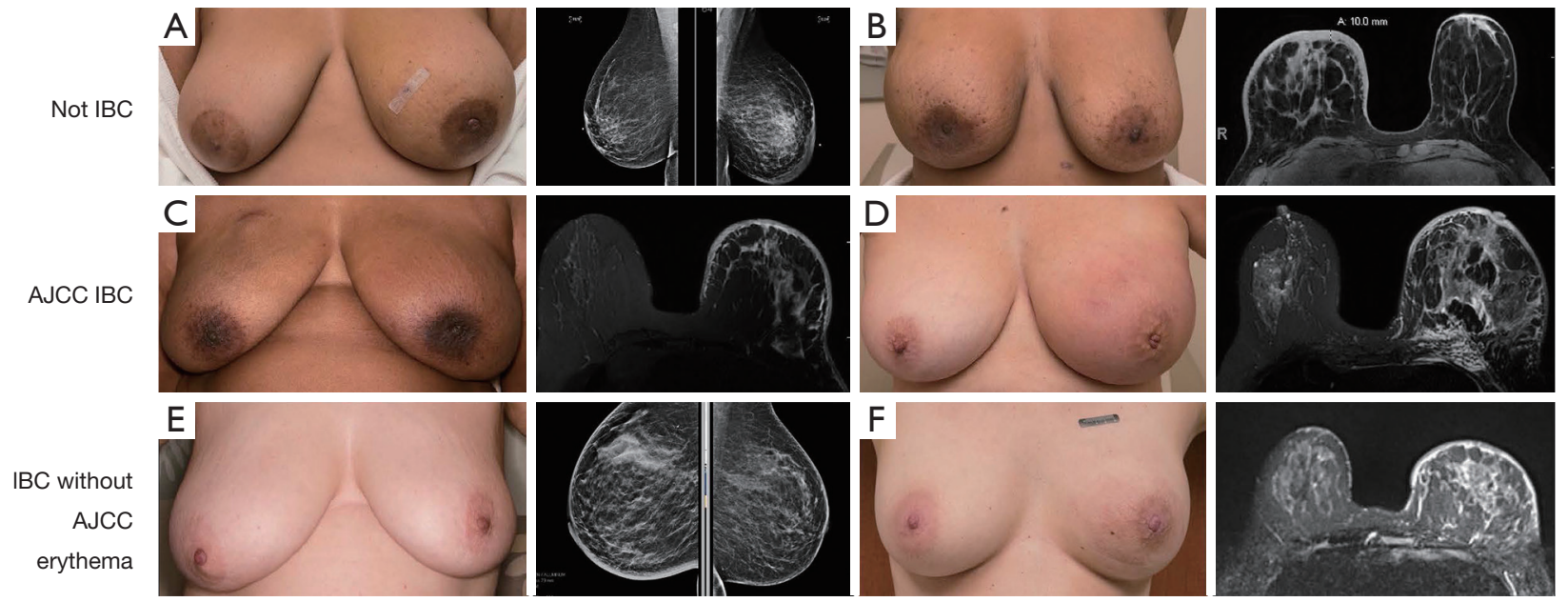

Figure 1 Variations in the presentation of malignant inflammatory breast highlight the difficulty in diagnosing inflammatory breast cancer (IBC). Medical photographs (left) and images demonstrating diffuse skin edema (right) are shown for 6 patients presenting for evaluation of breast skin symptoms to the MD Anderson Cancer Center IBC clinic. (A) Patient described 2 years of clear nipple discharge and 10 months of left breast swelling that progressed more rapidly in the last 2 months. At the time of evaluation, the left breast was larger than the right with diffuse skin edema apparent clinically and on imaging, and nipple inversion. The physical exam is consistent with IBC lacking frank erythema, but the timing exceeds the 6-month window. Invasive breast cancer was confirmed on biopsy and the disease was staged as T4B, non-inflammatory. (B) Patient described rapid onset of breast swelling and skin edema with non-erythematous color change and inverted nipple. Clinical exam is consistent with IBC, and imaging was suspicious for breast cancer. Biopsy demonstrated carcinoma of Mullerian origin; PET/CT confirmed metastatic ovarian cancer, not IBC. (C) Patient noted swelling, pain, and erythema in the left breast exactly 3 weeks before presentation. Imaging and biopsy confirmed invasive breast cancer, and the disease was staged as T4D, IBC. (D) Abrupt onset of a left breast mass and breast swelling followed by erythema. Imaging and biopsy confirmed invasive breast cancer and the disease was staged as T4D, IBC. (E) Abrupt onset of a right breast mass, global breast swelling, and subtle diffuse clinical skin edema, with diffuse skin edema on imaging. Erythema is not present. Biopsy confirmed breast cancer. Disease was staged as T4D, IBC. (F) Patient noted a left breast mass at 5 months postpartum. Mammogram was reportedly benign. The patient presented to her physician a month later with increased swelling and erythema and was diagnosed with mastitis. Two months later, when the photograph was obtained, sonography confirmed a mass, and biopsy revealed invasive cancer. Disease was staged T4D, IBC.

Speaking to the significance of this issue, an expert panel convened by the Susan G. Komen Foundation on IBC identified the lack of a more formal definition of IBC as the most pressing issue to be addressed to improve outcomes for IBC (11), and this panel is seeking to develop such a definition. In the meantime, both staging definitions as written require erythema, although many experts who treat large numbers of patients with IBC agree that this requirement should be removed from the staging system (11). Although some cases are characterized by diffuse erythema and overt skin edema or peau d'orange, significant variation at presentation leads to ambiguity in diagnosis (Figure 1). In The University of Texas MD Anderson Cancer Center's IBC clinic registry (12), redness was a component of presentation in only $69 \%$ of patients. Further, the criterion of one-third of the breast is nonspecific, not based on data, and not quantitative. Another variant of IBC described in the literature, occult IBC, involves having no clinical signs of IBC at all but the presence of dermal lymphatic emboli (13); however, this variant could result from localized skin invasion and not represent IBC biology. At this time, cases such as these are not diagnosed as IBC in our dedicated IBC clinic. Finally, consistent with the impression that global skin changes need not be red but nonetheless represent the biology of IBC, we recently reviewed the medical photographs of 245 patients with untreated IBC and found that an a priori-defined triad of breast swelling, global skin change (red or otherwise), and nipple change was present in 60 cases and was associated with significantly worse outcomes (7). The 10-year actuarial overall survival rate for patients with this triad was $29.7 \%$ vs. $57.2 \%$ for all others $(\mathrm{P}=0.001)$. 


\section{Differential diagnosis and work-up of inflammatory breast syndrome}

Other issues to consider are those that delay the diagnosis at presentation before a malignancy is identified. The differential diagnosis in a patient with signs or symptoms of inflammation of the breast (including redness, edema, pain, or swelling) without a diagnosis, i.e., those with "inflammatory breast" or "inflammatory breast syndrome," includes both lactation-related and non-puerperal causes, and very few reports have been published that define the incidence of malignancy among patients presenting with inflammatory breast symptoms aside from lactationrelated mastitis (14-17). This means that little literature is available to assist the generalist faced with a patient with an inflammatory breast to optimize the work-up and to distinguish malignant from non-malignant etiologies. The most common diagnosis causing inflammatory breast syndrome is infectious mastitis, which is most common during lactation and typically responds quickly to antibiotics. The crucial diagnosis to rule out is IBC, as IBC is always stage III at diagnosis and progresses rapidly to stage IV (incurable) breast cancer when the diagnosis is delayed $(15,16)$. Also important to recognize is that IBC can occur in pregnant and lactating women, making the diagnosis in such patients especially challenging.

The incidence of IBC is low among women with breast cancer, typically estimated at $2-3 \%$. Assuming an incidence of 125 breast cancer cases per 100,000 women, the estimated incidence of IBC is approximately $2-3$ cases per 100,000 women (18). Therefore, many generalists may never encounter IBC in their careers. According to the available references, the incidence of IBC among women with an inflamed breast ranges from $5 \%$ to $50 \%$, highlighting the fact that IBC may not be that uncommon among women presenting with an inflamed breast, particularly when the patient is not pregnant or postpartum $(17,19,20)$. Indeed, in one report of women presenting with inflammatory breast symptoms who were not pregnant or postpartum, the incidence of breast cancer was $50 \%$ (17). In that study, Dabi et al. reviewed the experience at a tertiary university hospital in France and identified 76 non-pregnant or postpartum women presenting with inflammatory breast (benign and malignant causes). Thirty-eight of these patients were diagnosed with IBC, underscoring the likelihood that a greater proportion of patients seen at a tertiary referral center would have cancer. The other 38 patients had benign diagnoses of infectious and inflammatory etiologies. Infectious etiologies were predominantly retroareolar abscess $(n=15$, $71.4 \%$ ), although galactoceles, infected cysts, and abscess associated with fibroadenoma and an abscess after posttraumatic fat necrosis were also observed. Inflammatory etiologies were periductal mastitis $(\mathrm{n}=5,29.4 \%)$, postirradiation mastitis $(\mathrm{n}=3,17.6 \%)$, granulomatous mastitis $(\mathrm{n}=2,11.8 \%)$, Mondor disease $(\mathrm{n}=1,5.9 \%)$, post-traumatic fat necrosis $(\mathrm{n}=1,5.9 \%)$, inflammatory adenofibroma $(\mathrm{n}=1,5.9 \%)$, or unclassified mastitis $(\mathrm{n}=4,23.5 \%)$. Dabi and colleagues proposed a diagnostic algorithm focused on the importance of identifying IBC when present and treating it appropriately as an oncologic emergency (17). An adaptation of their test-and-treat approach to diagnosing IBC in non-puerperal woman, incorporating guidance for pregnant or postpartum patients, is shown as Figure 2. In a smaller study of patients presenting with inflammatory breast symptoms, Froman et al. reported only one case of IBC among 22 patients presenting to a breast center with a red breast, with the predominant benign diagnosis being mastitis, abscess, radiation-induced erythema, cellulitis, and post-radiation morphea (19). Kamal et al. reviewed 197 cases of clinically diagnosed mastitis (used broadly to describe breast inflammation) and proposed grouping these cases as infectious, non-infectious/inflammatory, or malignant. They identified 11 cases of IBC (5.6\%), none in lactating women, and all in women who were older than 40 years (20). Collectively, these findings indicate a broad range in the incidence of IBC among patients presenting with a red or inflamed breast ( $4.5 \%$ to $50 \%)$. A weighted average using the data from the three studies described here is $16.9 \%$.

\section{Optimal imaging-and-biopsy approach to rule out IBC}

One commonality among IBC patients is a history that included evaluation with mammography that was reviewed as normal or no mass was identified. Indeed, in the study by Kamal et al., a review of images reveals that simple and malignant forms of mastitis have many signs in common (20). Mammographic signs were considered less discriminating than ultrasonography for identifying malignancy. Diffuse skin thickening and increased density favored malignant mastitis, whereas dilated retroareolar ducts and characteristic calcification patterns favored noninfectious forms. All of the patients with IBC had skin thickening on ultrasonography, but only $54 \%$ had skin thickening identified on mammography (20). However, 


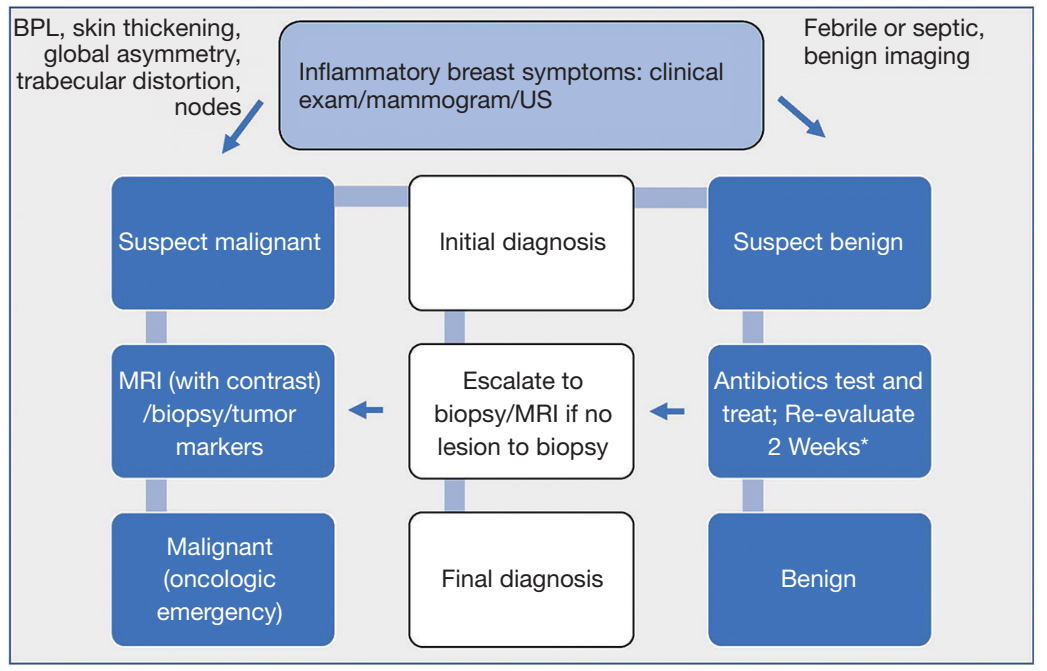

Figure 2 Non-lactating inflammatory breast algorithm [adapted from Dabi et al. 2017 (17)]. All non-lactating patients with inflammatory breast syndrome should have breast imaging, including ultrasonography. If malignancy is suspected but no identified lesion is amenable to biopsy, or if a "test and treat" strategy fails to resolve symptoms, magnetic resonance imaging (MRI) with biopsy is indicated. *, in lactating patients with strongly suspected acute mastitis, a reasonable approach is to begin with "test and treat" strategy and go back to the top of the algorithm if no marked improvement is noted after 2 weeks of antibiotics. Imaging could be obtained when the antibiotics are prescribed to ensure no delay if the symptoms progress or fail to resolve.

skin thickening can also be present in benign mastitis (Figure 3). In the study by Dabi et al., the presence of a mass (present in $21 / 45$ cases, malignant in $15 / 20, \mathrm{P}=0.04$ ), microcalcifications (present in 20/45 cases, malignant in $16 / 20, \mathrm{P}=0.04$ ) or focal asymmetry on mammography (present in $9 / 45$ cases, malignant in $8, \mathrm{P}<0.001$ ) were all significantly associated with malignant disease, but these signs were not present in all cases (17). Overall, relative to patients with benign disease, patients with a malignant lesion were more likely to have skin thickening $58 \%$ vs. $32 \%, \mathrm{P}=0.05$ ) and more lymph nodes suspected of harboring disease at clinical examination $(50 \%$ vs. $8 \%, \mathrm{P}<0.001)$. Patients with malignant disease were also significantly older $(\mathrm{P}=0.022)$ and had significantly larger palpable masses $(8.1$ vs. $4.1 \mathrm{~cm}, \mathrm{P}<0.001)$. Conversely, precise delimitation of the mass on ultrasonography was significantly associated with benign lesions. In work from Le-Petross et al. (21), primary breast lesions were more often visible on sonography than on mammography. An interesting sonographic feature best demonstrated on extended-field-of-view images was a linear infiltrative pattern that dissected through the breast parenchyma with loss of normal architecture (Figure 4). Critically, targeting this area of focal linear infiltration in the absence of a discrete mass on sonography yielded a diagnosis of cancer and enabled evaluation of biological markers in this study (21). Moreover, primary breast lesions on a background of extensive diffuse edema were more likely to be detected on MRI (when contrast is used) than on ultrasonography or mammography (Figure 5), and this additional information can assist in identifying a target for biopsy (21). Yang et al. reviewed and compared mammography, ultrasonography, MRI, and positron emission tomography/computed tomography (PET/ $\mathrm{CT}$ ) for their ability to identify breast parenchymal lesions, both to aid in the radiographic diagnosis and to guide clinicians regarding definitive biopsy (22). Among the 80 patients included in that study, 75 (94\%) had undergone mammography, 76 (95\%) sonography, 33 (41\%) MRI with contrast, and 24 (30\%) PET/CT. A primary parenchymal breast lesion was found in 60 patients $(80 \%)$ on mammography (mass or calcifications), 72 (95\%) on sonography (mass or architectural distortion), 23 (96\%) on PET/CT (hypermetabolic lesion), and 33 (100\%) on MRI (enhancing lesion). These findings highlight the value of MRI in the work-up of IBC to guide biopsy when earlier imaging has failed to reveal a dominant lesion or shows abnormal but inconclusive results. Notably, $97 \%$ of the women in this study had non-fatty breasts despite being largely postmenopausal. The authors speculate that the breast parenchymal background may have contributed to 

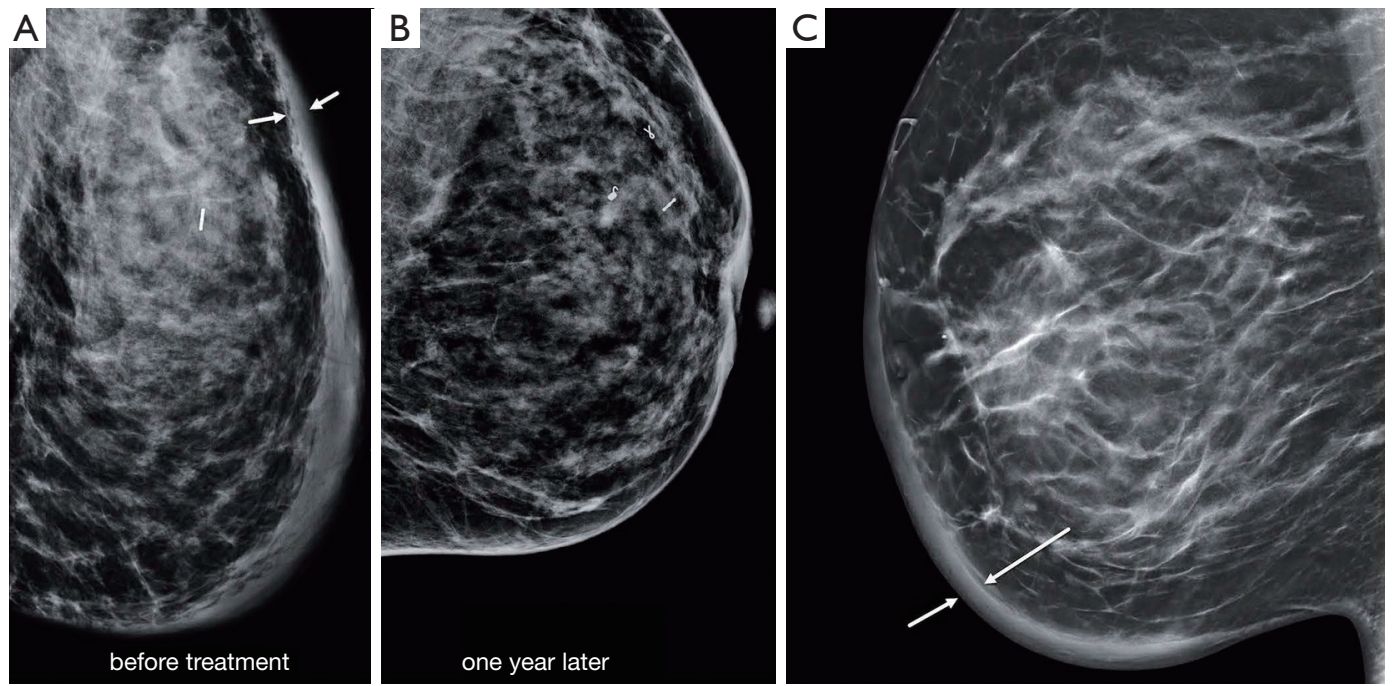

Figure 3 Skin edema in granulomatous mastitis. A 41-year-old woman with left breast pain, redness, and swelling over a few days that had not improved on ciprofloxacin and doxycycline. (A) Mammography at presentation revealed diffuse skin thickening with dense breast tissue in the superior breast. The patient subsequently had three biopsies (at initial presentation, 2 weeks, and 2 months later), all showing dense stromal fibrosis with acute and chronic inflammation and granulation tissue, all features suggestive of granulomatous mastitis. Left axilla lymph node biopsy was also negative for malignancy. The patient was treated with prednisone and methotrexate. (B) Follow-up at 2 years confirmed no cancer and improved left breast swelling and hardness. (C) For comparison, a mammogram of a 43-year-old woman with inflammatory breast carcinoma revealing diffuse skin thickening (arrows).

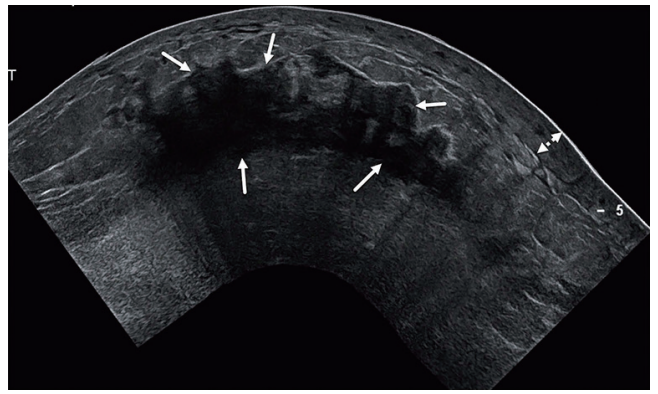

Figure 4 Breast ultrasonography with extended field of view. A 49-year-old woman with left breast and axillary swelling. Ultrasound-guided biopsy of the breast and axilla revealed poorly differentiated carcinoma positive for the estrogen and progesterone receptors and negative for Her2neu. The extended field of view ultrasound image revealed a large 9-cm area of abnormal tissue with posterior acoustic shadowing (white arrows). Associated skin thickening (2-headed arrows) was measured at $6 \mathrm{~mm}$ (normal skin thickness is $3 \mathrm{~mm}$ or less).

the poor visibility of a primary breast parenchymal lesion on mammography in 15 patients (20\%). Also, the basic imaging findings associated with IBC in that study agreed with those described by others (23-30), namely, diffusely increased parenchymal density (now termed global asymmetry according to the ACR BIRADS lexicon (31), trabecular distortion, and skin thickening on mammography. The same group undertook an expanded analysis focusing on MRI and identified a very high likelihood of detecting a breast parenchymal lesion with MRI (98\%, n=80) compared with $68 \%$ on mammography. Among patients with a breast parenchymal lesion, the most common MRI finding was a mass or multiple masses (57 of 78, 73\%) (22). Masses were often multiple, small, and confluent (47 of 57, 82\%); mass margins were irregular (43 of 57, 75\%); and had a heterogeneous internal enhancement pattern (47 of 57, $82 \%)$. Kinetic analysis revealed a delayed washout pattern in 66 of 78 tumors (85\%). MRI showed skin thickening in 74 of 80 breasts (93\%), whereas mammography showed skin thickening in 56 of 78 breasts (72\%) (22).

The edema component of IBC is well demonstrated on the T2-weighted images of a standard MRI examination (Figure 5), triggering some investigators to assess the value of this edema to distinguish benign inflammation from malignant inflammation or its predictive potential if the edema resolved. Kanao et al. retrospectively reviewed 162 patients presenting with inflammatory breast symptoms 

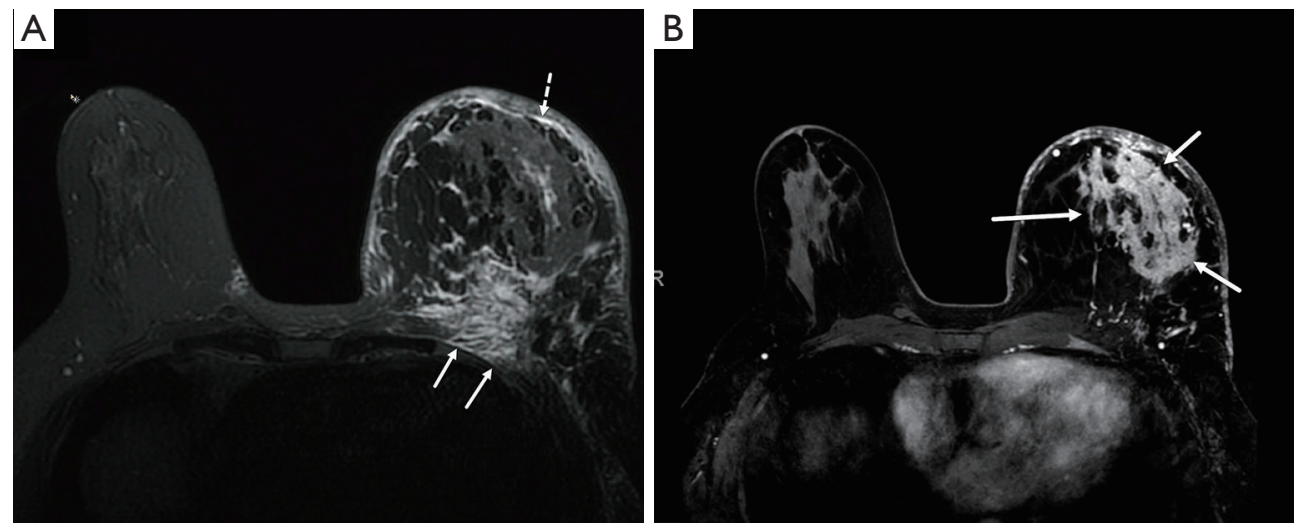

Figure 5 Non-contrast T2 image and contrast-enhanced T1 image show extensive edema around tumor in a 49-year-old woman with left breast inflammatory carcinoma. (A) The T2-weighted MR image shows diffuse high T2-signal or edema throughout the enlarged left breast, typical of IBC, noted in the subcutaneous region (broken arrow), throughout the breast, and in the pectoral or chest wall region (2 solid arrows). (B) The contrast-enhanced image shows the enhancing tumor (solid arrows), which appears dark on image A. This tumor bed can be masked on mammography or ultrasonography by the surrounding edematous tissue, since both edema and tumor would appear white on mammography.

who underwent breast MRI [T2-weighted imaging, with diffusion weighted imaging (DWI) or dynamic contrastenhanced (DCE) imaging] from 2008 through 2015. Among breast lesions with high-T2-signal intensity, 14 were benign inflammatory lesions, 69 were benign non-inflammatory lesions, 16 were malignant inflammatory lesions, and 63 were malignant non-inflammatory lesions (32). Notably, segmentation of those breast lesions having high T2 signals [by apparent diffusion coefficient (ADC) mapping, which is derived from T2-weighted images and DWI] could distinguish benign from malignant inflammatory conditions, but ADC mapping of low-T2-signal areas did not (32). In another evaluation of MRI, Harada et al. evaluated a model of breast edema scores in 408 women with breast cancer and found that the edema detectable on T2-weighted images was related to prognosis after neoadjuvant chemotherapy (13).

These findings hold promise for the development of a non-contrast breast MRI exam that relies on T2-weighted imaging and DWI without DCE, which would reduce concerns about nephrogenic systemic fibrosis or deposition of gadolinium-based contrast agents in tissue; however, further research with larger numbers of patients is needed. When this review was written, breast MRI must be done with intravenous contrast to be effective (22). Indeed, in our own experience at a dedicated IBC clinic, we identified one case where use of MRI without contrast for an implant rupture led to delay in the diagnosis of IBC.

\section{Conclusions}

IBC is an oncologic emergency, and making the diagnosis promptly can be the difference between curable and incurable disease. Understanding how to make this difficult diagnosis can save lives. Although IBC is relatively uncommon, at one tertiary referral center for breast symptoms, it represented $50 \%$ of patients who presented with inflammatory breast symptoms (17), so a high index of suspicion and immediate imaging workup is not excessive. Dedicated IBC clinics operating in the United States are willing to discuss complicated diagnostic cases with colleagues. Our recommendation, based on the literature reviewed, is that any patient with presumed benign mastitis that does not rapidly resolve with recommended therapy for benign disease should undergo breast imaging with mammography and ultrasonography, followed by MRI if available, and biopsy. Several studies have highlighted the limitations of mammography and, to a lesser degree, ultrasonography for identifying IBC with high specificity; however, abnormal findings on these exams should prompt further work-up, and even if MRI is not available, ultrasonography adds important diagnostic information over mammography alone (20-22). Notably, several groups have reported cases in which experienced breast oncology specialists diagnosed IBC that failed to meet AJCC or international consensus definitions because of the absence of erythema or the lack of concurrent breast 
edema and peau d'orange $(7,8,12)$. We assert that some IBC patients will not have frank erythema; however, compelling non-erythematous skin changes such as peau d'orange or other skin discolorations due to diffuse disease should be diagnosed as IBC, and patients should be treated with trimodality therapy including neoadjuvant systemic agents, and referral to a dedicated center for enrollment in an IBCspecific trial should be strongly encouraged.

The rarity of IBC and the lack of available information to fully quantify the number of women who present with inflammatory breast syndrome in terms of age and pregnancy status undoubtedly limit our understanding of the true incidence of IBC in women with symptoms. Further, the lack of objective pathobiological diagnostic criteria means that there is no "gold standard" against which to test assertions about clinical symptoms. Nevertheless, the information that is available affords a useful algorithm, and as many experts agree, revision of the staging criteria may be in order.

\section{Acknowledgments}

Funding: This work was supported by, State of Texas Grant for Rare and Aggressive Breast Cancers; the Susan G. Komen Training Grant for Reducing Breast Cancer Disparities (GTDR17498270); Cancer Center Support (Core) Grant P30 CA016672 from the National Cancer Institute, National Institutes of Health, to The University of Texas MD Anderson Cancer Center.

\section{Footnote}

Provenance and Peer Review: This article was commissioned by the Guest Editors (Naoto Ueno and Angela Alexander) for the series "Inflammatory Breast Cancer" published in Chinese Clinical Oncology. The article has undergone external peer review.

Reporting Checklist: The authors have completed the Narrative Review reporting checklist. Available at https:// dx.doi.org/10.21037/cco-21-116

Conflicts of Interest: All authors have completed the ICMJE uniform disclosure form (available at https://dx.doi. org/10.21037/cco-21-116). The series "Inflammatory Breast Cancer" was commissioned by the editorial office without any funding or sponsorship. Dr. WAW declares grant support from the National Institute of Health and Susan G. Komen, as well as the State of Texas. She has received honoraria or fees from Exact Science, Epic Science, and IBC East and West, and holds scientific leadership positions with the American Society for Radiation Oncology and San Antonio Breast Cancer Symposium. The authors have no other conflicts of interest to declare.

Ethical Statement: The authors are accountable for all aspects of the work in ensuring that questions related to the accuracy or integrity of any part of the work are appropriately investigated and resolved. With regard to patient information that has not been previously reported, this study was conducted in accordance with the Declaration of Helsinki (as revised in 2013) and was approved by institutional/regional/national ethics/committee/ethics boards of The University of Texs MD Anderson Cancer Center.

Open Access Statement: This is an Open Access article distributed in accordance with the Creative Commons Attribution-NonCommercial-NoDerivs 4.0 International License (CC BY-NC-ND 4.0), which permits the noncommercial replication and distribution of the article with the strict proviso that no changes or edits are made and the original work is properly cited (including links to both the formal publication through the relevant DOI and the license). See: https://creativecommons.org/licenses/by-nc-nd/4.0/.

\section{References}

1. Bonnier P, Charpin C, Lejeune C, et al. Inflammatory carcinomas of the breast: a clinical, pathological, or a clinical and pathological definition? Int J Cancer 1995;62:382-5.

2. Hirko KA, Soliman AS, Banerjee M, et al. A comparison of criteria to identify inflammatory breast cancer cases from medical records and the Surveillance, Epidemiology and End Results data base, 2007-2009. Breast J 2014;20:185-91.

3. Woodward WA. Inflammatory breast cancer: unique biological and therapeutic considerations. Lancet Oncol 2015;16:e568-76.

4. Hance KW, Anderson WF, Devesa SS, et al. Trends in inflammatory breast carcinoma incidence and survival: the surveillance, epidemiology, and end results program at the National Cancer Institute. J Natl Cancer Inst 
2005;97:966-75.

5. Ueno NT, Espinosa Fernandez JR, Cristofanilli M, et al. International Consensus on the Clinical Management of Inflammatory Breast Cancer from the Morgan Welch Inflammatory Breast Cancer Research Program 10th Anniversary Conference. J Cancer 2018;9:1437-47.

6. Kalli S, Semine A, Cohen S, et al. American Joint Committee on Cancer's Staging System for Breast Cancer, Eighth Edition: What the Radiologist Needs to Know. Radiographics 2018;38:1921-33.

7. Balema $W$, Liu D, Shen $Y$, et al. Inflammatory breast cancer appearance at presentation is associated with overall survival. Cancer Med 2021;10:6261-72.

8. Schairer C, Hablas A, Eldein IAS, et al. Clinicopathologic and mammographic characteristics of inflammatory and non-inflammatory breast cancer at six centers in North Africa. Breast Cancer Res Treat 2019;176:407-17.

9. Lê MG, Arriagada R, Contesso G, et al. Dermal lymphatic emboli in inflammatory and noninflammatory breast cancer: a French-Tunisian joint study in 337 patients. Clin Breast Cancer 2005;6:439-45.

10. Amin MB, Edge SB. editors. AJCC Cancer Staging Manual, 8th edition. NY: Springer, 2017.

11. Mason G, Overmoyer B, Woodward W, Badve S, Schneider R, Jagsi R, Lang JE, Alpaugh M, Smith K, Milleret K. Abstract P6-15-03: Inflammatory breast cancer (IBC) defined: Proposed common diagnostic criteria and scoring - Moving beyond the subjective 'clinical diagnosis' of IBC to advance research. Cancer Res 2020;(80):P6-15-03.

12. Fouad TM, Ueno NT, Yu RK, et al. Distinct epidemiological profiles associated with inflammatory breast cancer (IBC): A comprehensive analysis of the IBC registry at The University of Texas MD Anderson Cancer Center. PLoS One 2018;13:e0204372.

13. Harada TL, Uematsu T, Nakashima K, et al. Evaluation of Breast Edema Findings at T2-weighted Breast MRI Is Useful for Diagnosing Occult Inflammatory Breast Cancer and Can Predict Prognosis after Neoadjuvant Chemotherapy. Radiology 2021;299:53-62.

14. Lucas FV, Perez-Mesa C. Inflammatory carcinoma of the breast. Cancer 1978;41:1595-605.

15. Resetkova E. Pathologic aspects of inflammatory breast carcinoma: part 1. Histomorphology and differential diagnosis. Semin Oncol 2008;35:25-32.

16. Hester RH, Hortobagyi GN, Lim B. Inflammatory breast cancer: early recognition and diagnosis is critical. Am J
Obstet Gynecol 2021;225:392-6.

17. Dabi Y, Darrigues L, Pons K, et al. Incidence of inflammatory breast cancer in patients with clinical inflammatory breast symptoms. PLoS One 2017;12:e0189385.

18. KFF analysis of United States Cancer Statistics Incidence: 1999 - 2017, WONDER Online Database. United States Department of Health and Human Services, Centers for Disease Control and Prevention and National Cancer Institute; 2020. Available online: https://wonder.cdc.gov/cancer-v2017.HTML on March $5,2021$.

19. Froman J, Landercasper J, Ellis R, et al. Red breast as a presenting complaint at a breast center: an institutional review. Surgery 2011;149:813-9.

20. Kamal RM, Hamed ST, Salem DS. Classification of inflammatory breast disorders and step by step diagnosis. Breast J 2009;15:367-80.

21. Le-Petross HT, Cristofanilli M, Carkaci S, et al. MRI features of inflammatory breast cancer. AJR Am J Roentgenol 2011;197:W769-76.

22. Yang WT, Le-Petross HT, Macapinlac H, et al. Inflammatory breast cancer: PET/CT, MRI, mammography, and sonography findings. Breast Cancer Res Treat 2008;109:417-26.

23. Dershaw DD, Moore MP, Liberman L, et al. Inflammatory breast carcinoma: mammographic findings. Radiology 1994;190:831-4.

24. Droulias CA, Sewell CW, Mcsweeney MB, et al. Inflammatory carcinoma of the breast: A correlation of clinical, radiologic and pathogic findings. Ann Surg 1976;184:217-22.

25. Günhan-Bilgen I, Ustün EE, Memiş A. Inflammatory breast carcinoma: mammographic, ultrasonographic, clinical, and pathologic findings in 142 cases. Radiology 2002;223:829-38.

26. Kushwaha AC, Whitman GJ, Stelling CB, et al. Primary inflammatory carcinoma of the breast: retrospective review of mammographic findings. AJR Am J Roentgenol 2000;174:535-8.

27. Caumo F, Gaioni MB, Bonetti F, et al. Occult inflammatory breast cancer: review of clinical, mammographic, US and pathologic signs. Radiol Med 2005;109:308-20.

28. Tardivon AA, Viala J, Corvellec Rudelli A, et al. Mammographic patterns of inflammatory breast carcinoma: a retrospective study of 92 cases. Eur J Radiol 1997;24:124-30.

29. Lee KW, Chung SY, Yang I, et al. Inflammatory breast cancer: imaging findings. Clin Imaging 2005;29:22-5. 
30. Chow CK. Imaging in inflammatory breast carcinoma. Breast Dis 2005-2006;22:45-54.

31. American College of Radiology ACR BI-RADS Mammography. In: ACR breast imaging reporting and data system, breast imaging atlas. American College of

Cite this article as: Le-Petross HT, Balema W, Woodward WA. Why diagnosing inflammatory breast cancer is hard and how to overcome the challenges: a narrative review. Chin Clin Oncol 2021;10(6):58. doi: 10.21037/cco-21-116
Radiology, Reston, VA, 2003.

32. Kanao S, Kataoka M, Iima M, et al. Differentiating benign and malignant inflammatory breast lesions: Value of T2 weighted and diffusion weighted MR images. Magn Reson Imaging 2018;50:38-44. 